\title{
THE DOMINANT INTERACTIONS BETWEEN SNR AND THE ISM
}

\author{
Robert Braun \\ National Radio Astronomy Observatory*, \\ P.O. Box O, Socorro, New Mexico 87801, USA
}

\begin{abstract}
The interactions of supernovae ejecta with the surrounding medium which have a dominant influence on the resulting SNR dynamics and brightness are considered for both young and evolved objects. The important roles of clumpy ejecta in determining the brightness of young SNR and of shock impingement on a pre-existing shell in influencing both the brightness and subsequent dynamics of evolved SNR are stressed.
\end{abstract}

Introduction: The basic types of interactions which might be expected to occur between the ejecta of a supernova and the surrounding environment have been postulated for some time (e.g. McKee 1983). These are: the interaction of diffuse ejecta with 1) diffuse ISM/CSM either with or without a power law density profile, 2$)$ high density clumps ( $\leq .1 \mathrm{pc})$ of ISM/CSM, 3$)$ high density clouds ( $\geq 1 \mathrm{pc}$ ) of ISM and 4) high density walls of a pre-existing cavity, as well as the interaction of clumpy ejecta with each of the preceding components. The relatively short timescales for the dissipation and/or thermalization of clumpy ejecta make it more probable that only its interaction with 5) diffuse shocked ejecta/ISM/CSM may be commonly observed. While all of these processes are likely to occur to a greater or lesser extent, it is interesting to consider which might play a dominant role in the development of observable (shell-type) remnants.

Dominant Interactions in Young SNR: The dynamics of the youngest known galactic SNR appear to be governed primarily by process 1) above. The diffuse components remain dynamically coupled and generate a classical spherical shock front albeit with reverse shock propagation back through the ejecta. The slow moving optical knots seen in Cas A and Kepler represent interactions of type 2) above, while evidence for interstellar clouds (process 3 ) is seen in the Balmer line filaments of Tycho and SN1006. However, the mass of diffuse ejecta that is involved in powering the shock front appears to be surprisingly low. IRAS observations have allowed a good estimate of the shocked ISM mass to be made in the case of Tycho and (perhaps with somewhat higher uncertainty due to a possibly abnormal gas/dust ratio) Cas A (Braun 1987, Dwek 1987), while current expansion measurements have allowed determination of the dynamical age. Together these quantities imply a diffuse ejecta mass of about $0.4 \mathrm{M}_{\odot}$ with an associated SN energy, $\mathrm{E}_{o} \leq 2 \times 10^{50} \mathrm{erg}$ for both SNR. These values imply that most of the ejecta mass in these systems, $\sim 1 \mathrm{M}_{\odot}$ in Tycho and $\geq 5 \mathrm{M}_{\odot}$ in Cas $A$, resides in clumps. Furthermore, since the shock velocity has now decreased from an initial value of $\sim 10,000 \mathrm{~km} \mathrm{~s}^{-1}$ to $\sim 2,000 \mathrm{~km} \mathrm{~s}^{-1}$, the remaining ejecta mass will contribute only $1 / 25$ the energy per unit mass of the fast diffuse component. Total energies are thus likely to be $\leq 3 \mathrm{x}$ $10^{50} \mathrm{erg}$. Observational evidence for clumpy ejecta is seen in the bright clumpy radio/X-ray ring interior to the outer shock in Tycho (e.g. Seward, Gorenstein and Tucker 1983) which dominates the emission from this source. Even more direct evidence for clumpy ejecta is seen in Cas A where both high-density clumps, the optically observed FMK (e.g. Van den Bergh and Kamper 1983), and more tenuous clumps, the bow-shock producing blobs which dominate the radio structure (Braun, Gull and Perley 1987), are seen continuously puncturing the shell of decelerated diffuse ejecta and shocked ISM.

It seems that the dynamics of young SNR are dominated by diffuse interactions of type 1) and to a lesser extent type 3), and it is these processes which are responsible for Balmer line optical filaments and collisionally heated dust emission. High brightness X-ray and radio emission (and high velocity oxygen line emission) on the other hand is closely tied to the secondary shocks

* The National Radio Astronomy Observatory is operated by Associated Universities Inc., under contract with the National Science Foundation. 
and turbulence caused by the interception of clumpy ejecta (wherein the majority of the pre-cursor mass resides) by the decelerated reverse/outer shock zone. Dominant Interactions in Evolved SNR: By the time a supernova remnant has become at least partially radiative the effects of clumpy ejecta should no longer play a dominant role in the source's emission and evolution. Clumps with both high density and velocity will have deposited their energy in the SN environment after shell penetration, while lower density and/or velocity clumps will have been ablated or evaporated in the SNR interior, adding their energy to that driving the diffuse shell. The radiative shell of optical filaments surrounding such objects has traditionally been interpreted as the result of the shock overrunning randomly occuring clouds in the $\mathrm{SN}$ environment. This interpretation no longer seems to be viable.

Individual cases that have been studied in detail have shown the existence of cold, unshocked shells and cavity walls which must pre-date the SN event. These structures are illustrated in figure 1 , where the warm shock-heated dust emission from IC 443 centered at $\alpha, \delta=$ $6^{h} 14^{m}, 22 .^{\circ} 5$ has a dense unshocked shell of cool dust immediately to the west and south, and in figure 2, where much of the western and northern rim of the Cygnus Loop is seen to be abutting a region of higher density traced by the cool dust distribution. (The observations illustrated in these figures are discussed in Braun and Strom 1986a,b.) In IC 443 the shock has already been propagating within the high density gas sufficiently long that shock-accelerated molecular and atomic gas (e.g. Braun and Strom 1986a) are seen in profusion. In the Cygnus Loop on the other hand, this interaction is only beginning, as evidenced by the high cavity wall densities $\left(n_{H} \geq 100 \mathrm{~cm}^{-3}\right)$ but non-detection of shocked $\mathrm{CO}$ and only faint accelerated HI (Strom, priv.com.). The kinetic energy implied by the gas mass and velocity within a shell geometry in these two cases, $\mathrm{E}_{k}{ }^{I S M}=0.55 \times 10^{50} \mathrm{erg}$ corre-
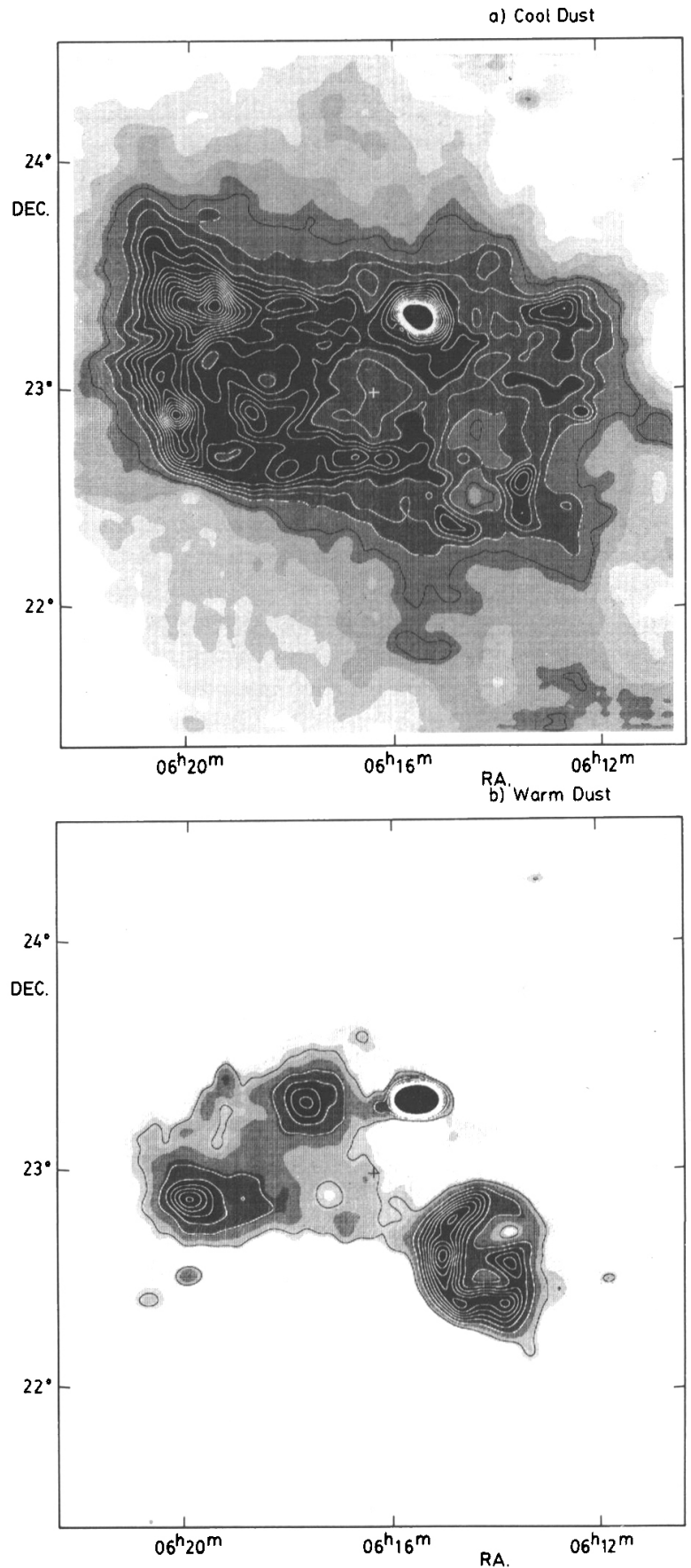

Fig. 1a and b. Cool and warm dust emission components within the IC 443 complex derived from images in the four IRAS bands by a three-component spectral decomposition. The shock-heated dust of the IC $443 \mathrm{SNR}$ is centered near $\alpha, \delta=$ $6^{h} 14^{m}, 22 .^{\circ} 5$. Note the cool high-density shell immediately outside the shocked gas to the south and west. 
sponds to a total energy $\mathbf{E}_{o}=2 \times 10^{50}$ erg assuming the $28 \%$ lower limit to the kinetic energy applicable to (post-) Sedov evolution. While few SNR have been studied carefully enough to detect unshocked shells, shock-accelerated HI has been found in every evolved SNR studied to date with sufficient sensitivity, IC 443, VRO 42.05.01, CTB 109, G78.2+2.1 (Braun and Strom 1986c) and OA184 (Routledge et.al. 1986). Consideration of the conditions required to detect accelerated $\mathrm{HI}$ implies columns of $n_{H} \cdot t \geq 3 \cdot 10^{5} \mathrm{~cm}^{-3} \mathrm{yr}$ shocked at $100 \mathrm{~km}$ $\mathrm{s}^{-1}$ (c.f. Braun and Strom 1986a). Since accelerated $\mathrm{HI}$ is found over a significant fraction of the shell, but yet a hard X-ray interior (detected so far in IC 443, the Cygnus Loop, CTB 109 and G78.2+2.1) indicates much higher shock velocities in the recent past, a shell geometry for this high density gas is strongly implied.

A further indication for the common occurrence of a pre-existing cavity is given by consideration of the implied initial energies of SNR obtained by various means. As indicated above, total energy estimates based on IR determined masses and source dynamics for Tycho, a type I SNR, and Cas A, presumably a type Ib or II SNR, are quite well determined, $\mathrm{E}_{o} \leq 3 \times 10^{50}$. Similar total energies, $\mathrm{E}_{o}=2 \times 10^{50} \mathrm{erg}$, are implied above by considering the kinetic energy within a shell geometry for IC 443 and the Cygnus Loop, the evolved remnants of apparently massive SN. The analysis of eight $\mathrm{X}$-ray detected SNR in the LMC by Long and Helfand (1979) assuming a Sedov description of the X-ray emitting gas results in a consistent mean initial energy estimate $\mathbf{E}_{o}=2 \times 10^{50} \mathrm{erg}$. This consistency disappears when considering the initial energies implied by assuming a Sedov description of the pressure driving the shock into the radiatively cooling gas observed optically (e.g. Dopita 1979; Blair, Kirshner and Chevalier 1981). Implied energies derived from this method increase monotonically with SNR diameter between a few times $10^{49}$ erg at 5 pc and $10^{52}$ erg at 100 pc. It seems that the epoch of radiative cooling in SNR is accompanied by a radical departure from adiabatic shock propagation (like the transition from inner cavity to cavity wall shocks) rather than merely a perturbation resulting from randomly distributed clouds within a more tenuous substrate. 
More General Considerations: It has too often been the case that supernova remnants were considered in isolation. While the remnants of type I SN may have the oppportunity of expanding into a relatively pristine environment (and as a consequence rapidly dissipate their energy in an unobtrusive manner after the first few hundred years of clumpy ejecta interception), the remnants of type II and probably type Ib SN must almost certainly encounter an environment which has been heavily processed by both the progenitor and possibly other association members. Such a scenario is graphically illustrated in figure 3 (from Braun and Strom 1986a), where the shock from the IC $443 \mathrm{SN}$ is seen to propagate not only in the cavity of the precursor, but into the adjoining cavity surrounding at least one wellidentified fellow association member.

The recent work of Heiles (1987) gives a refreshing analysis of the ISM that takes into account the spatial and temporal distribution of SN I and SN II. The major difficulty that this analysis has in confronting observations is the high predicted value of the porosity parameter, implying an inordinately high filling factor for the hot ionized medium (HIM).

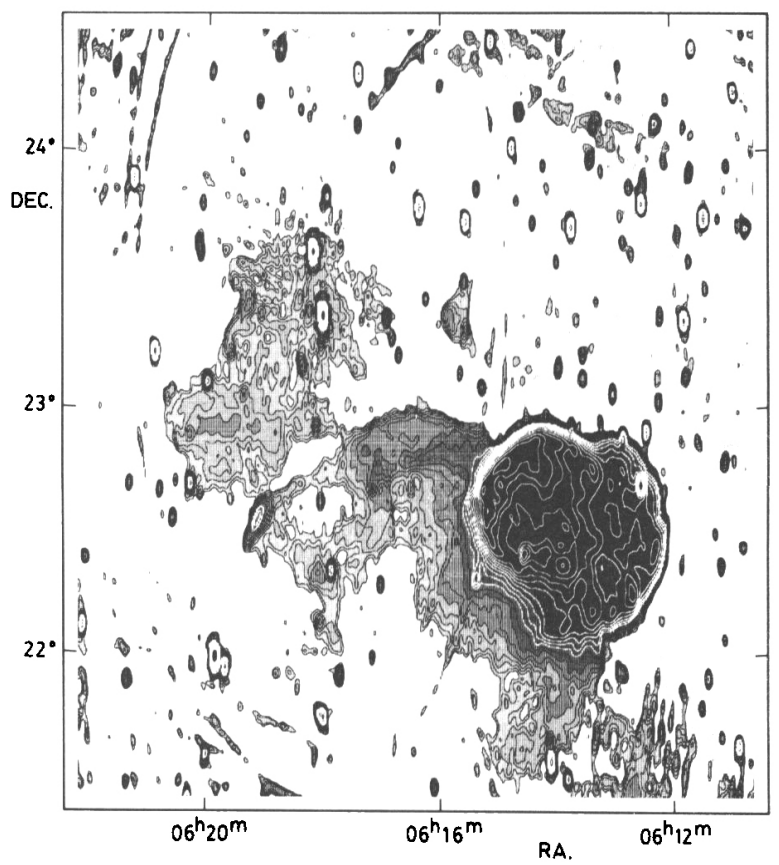

Fig. 3. Radio continuum emission from the IC 443 complex at $327 \mathrm{MHz}$. Thermal radio emission is confined to the diffuse region between $\alpha=6^{h} 18^{m}-6^{h} 20^{m}, \delta=22 .^{\circ} 5-23 .^{\circ} 5$. Non-thermal emission is seen from the bright shell centered near $\alpha, \delta=6^{h} 14^{m}, 22 .^{\circ} 5$ with radius $\sim 0 .{ }^{\circ} 4$, as well as the fainter interconnected shell centered near $\alpha, \delta=6^{h} 16^{m}, 22 .^{\circ} 2$ with radius $\sim 0{ }^{\circ} 8$ containing the B0.5 V star HD44139.

As Heiles suggests, the most straightforward way out of this dilemma is to adopt an SN energy smaller than the oft-quoted but poorly established $10^{51} \mathrm{erg}$ he assumes. In light of the preceding discussion this seems to be amply justified, and adopting $\mathrm{E}_{o}=2 \times 10^{50}$ erg gives HIM filling factors which are in reasonable agreement with those observed.

Ultimately, a better understanding of the makeup and energetic balance of a normal galaxy should emerge from the comprehensive study of our nearby neighbours M31 and M33. Both galaxies are currently being subjected to such an extensive observational assault that a much clearer view of the complex inierplay of processes which mold their media should soon be forthcoming.

\section{References:}

Braun, R. and Strom, R.G. 1986a, Astr. Ap., 164, 193-207

Braun, R. and Strom, R.G. 1986b, Astr. Ap., 164, 208-217

Braun, R. and Strom, R.G. 1986c, Astr. Ap. Suppl. 63, 345--401

Braun, R. 1987, Astr. Ap., 171, 233-251

Braun, R., Gull, S.F. and Perley R.A. 1987, Nature 327,395-398

Blair, W.P., Kirshner, R.P. and Chevalier, R.A. 1981, Ap. J., 247, 879-893

Dopita, M.A. 1979, Ap. J. Suppl. 40, 455-474

Dwek, E. 1987, in prep.

Heiles, C. 1987, Ap. J., 315, 555-566

Long, K.S. and Helfand, D.J. 1979, Ap. J. (Letters), 234, L77-L81

MKee, C.F. 1983, in Supernova Remnants and their X-ray Emission, eds. Danziger, I.J. and Gorenstein, P., Reidel, Dordrecht, 87-97

Routledge, D., Landecker, T.L. and Vaneldik, J.F. 1986, M.N.R.A.S., 221, $809-821$ 